\title{
Transconductance increase due to charge trapping during hot-carrier stress of nMOSFETs
}

\author{
J. M. Rafí and F. Campabadal \\ Institut de Microelectrònica de Barcelona, CNM-CSIC \\ Campus Universitat Autònoma de Barcelona, 08193 Bellaterra (Barcelona), Spain \\ E-mail: joanmarc.rafi@cnm.es
}

\begin{abstract}
An unusual hot-carrier degradation mode characterised by a transconductance increase during hot-carrier ageing of nMOS transistors is analysed. By measuring the effects of hot-carrier stress on drain and substrate characteristics and applying alternate static injection phases performed at different gate regimes, it is proved that the degradation is mainly due to negative charge trapping in a localised region near the drain. The transconductance increase is explained in terms of an exchange of the dominant role between the damaged and undamaged portions of the channel. This model is fully corroborated by $2 D$ device electric simulation results.
\end{abstract}

\section{Introduction}

In the past two decades, a lot of works have focused on the hot-carrier degradation of conventional nMOS transistors. The damage is commonly believed to result in charge trapping in the gate oxide [1] and interface-state generation at the $\mathrm{Si}_{-} \mathrm{SiO}_{2}$ interface $[2,3,4]$. The simplest approach states that the first is responsible for a parallel shift of the full $I_{d}$ vs. $V_{g}$ curves, while the second leads to subthreshold slope changes and transconductance decrease due to electron mobility degradation [5]. However, the specific particularities involving the nature, extension and position of the generated damage make physical understanding of hot-carrier degradation a rather complex subject.

In this paper, an unusual hot-carrier degradation mode characterised by a transconductance increase during hot-carrier stressing is analysed. In fact, a transconductance increase has already been predicted by a few simulation works [6,7], in which the presence of negative oxide trapped charges localised close to the drain junction has been found to result in this phenomenon. However, to our knowledge, this was neither corroborated by experimental results nor have the mechanisms giving rise to the phenomenon itself been fully analysed. A transconductance increase after long-term hot-carrier degradation has also been observed in case of hole trapping under low gate voltage stresses $[8,9]$ or in devices with an extremely high substrate impurity concentration $\left(\geq 10^{18} \mathrm{~cm}^{-3}\right)$ [10].

In contrast to the above experimental works, all of them performed under very special electric or technological conditions, the hot-carrier ageingmode analysed in the present paper has been observed in nMOS transistors of a conventional CMOS twin-tub process, stressed at maximum substrate current condition, and it has been directly related to the presence of negative trapped charges. 


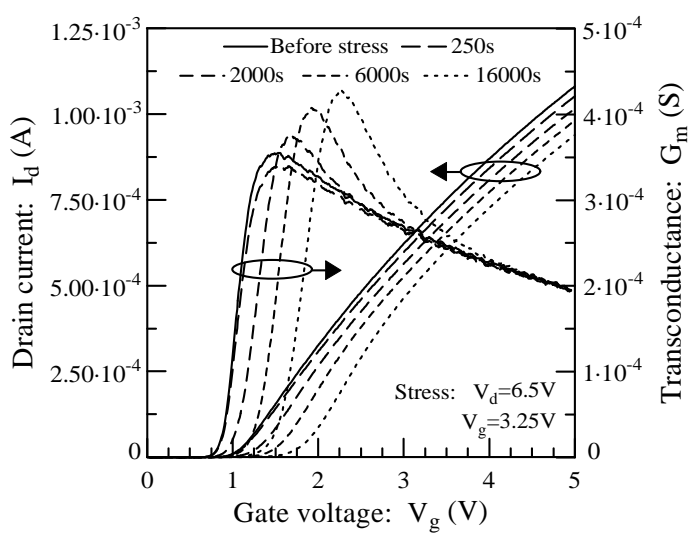

Fig. 1. $\mathrm{I}_{\mathrm{d}}$ vs. $\mathrm{V}_{\mathrm{g}}\left(\mathrm{V}_{\mathrm{d}}=0.1 \mathrm{~V}\right)$ curves and corresponding transconductance measured at various times of ageing.

\section{Devices and experimental procedure}

The n-channel devices used in this study were fabricated using a conventional twin-tub CMOS process, with a Doubly Doped Drain (DDD) structure. The nMOSFET dimensions were $\mathrm{L}_{\text {eff }}=1.3 \mu \mathrm{m}$ and $\mathrm{W}=75 \mu \mathrm{m}$, with a $\mathrm{SiO}_{2}$ gate oxide thickness of $25 \mathrm{~nm}$.

Previous to the study of device degradation, an electrical characterisation was carried out, evaluating the most representative aspects of drain $\left(I_{d}\right)$ and substrate current $\left(I_{\text {sub }}\right)$ characteristics. The gate voltage condition leading to maximum substrate current $\left(\mathrm{I}_{\text {submax }}\right.$ ) was determined to be at $\mathrm{V}_{\mathrm{g}}=\mathrm{V}_{\mathrm{d}} / 2$.

For the hot-carrier degradation analysis, the transistors were stressed at constant-voltage stress, with drain voltage $\left(\mathrm{V}_{\mathrm{d}}\right)$ ranging from 5.5 to $7 \mathrm{~V}$ and at the $\mathrm{I}_{\text {submax }}$ condition, which was experimentally found to give rise to the most severe degradation. The effect of the stress on the devices was mainly evaluated through the analysis of $I_{d}$ vs. $V_{g}$ curves, which were regularly measured at $\mathrm{V}_{\mathrm{d}}=0.1 \mathrm{~V}$ between different stress phases.

Threshold voltage $\left(\mathrm{V}_{\mathrm{th}} \equiv \mathrm{V}_{\mathrm{g}} @ \mathrm{I}_{\mathrm{d}}=0.01 \mathrm{~W} / \mathrm{L} \mu \mathrm{A}\right)$, maximum transconductance $\left(\mathrm{G}_{\mathrm{mmax}}\right)$ and linear drain current measured at $\mathrm{V}_{\mathrm{g}}=5 \mathrm{~V}\left(\mathrm{I}_{\mathrm{dlin}}\right)$ were the primary degradation monitors, all of them obtained from the $I_{d}$ vs. $V_{g}$ curves.

The fully automated experimental set-up used for both, the electrical characterisation and the stress experiments, consisted of an HP 4142B modular DC source/monitor, an HP 4085A switching matrix and a Wentworth automatic wafer prober. All experiments were carried out in a light-proof and electrically shielded probe station.

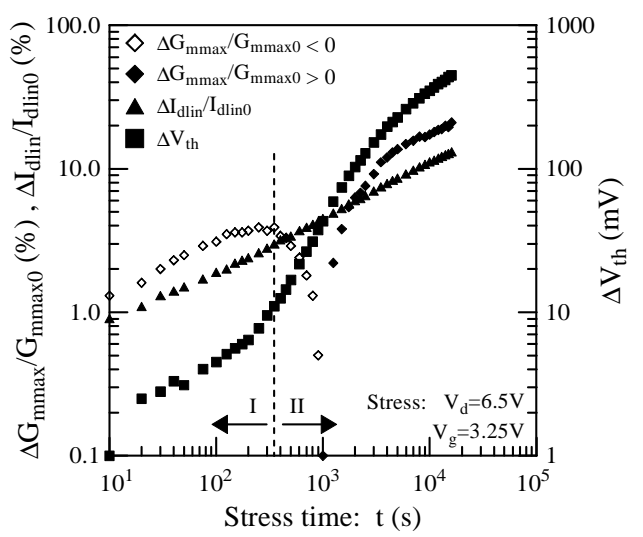

Fig. 2. $G_{\operatorname{mmax}}$ and $I_{\text {dlin }}$ relative degradation, together with $\Delta \mathrm{V}_{\mathrm{th}}$, as a function of stress time.

\section{Device degradation}

\section{$3.1 I_{d} v s . V_{g}$ and transconductance increase}

Fig. 1 shows typical $I_{d}$ vs. $V_{g}$ measured curves for an n-channel MOSFET subjected to hot-carrier stress. The degradation observed over the whole range of stress voltages is characterised by two main phases along the stress time, which have been found to be accelerated as stress voltages are increased or, alternatively, as channel lengths are reduced.

As shown in Fig. 2, the first phase (I in Fig. 2) is characterised by a weak decrease of device maximum transconductance $\left(\mathrm{G}_{\mathrm{mmax}}\right)$, together with a slight but continuous decrease of $\mathrm{I}_{\mathrm{dlin}}$ and $\mathrm{a}$ negligible positive threshold voltage shift $\left(\Delta \mathrm{V}_{\text {th }}\right)$.

As the stress progresses, the device enters a second phase (II in Fig. 2), in which there is a significant positive $\mathrm{V}_{\mathrm{g}}$ shift of the curves in their entirety as a function of increasing stress time, together with a gradual degradation in subthreshold slope and an unusual turn around in the transconductance behaviour, which now starts to rise, eventually reaching higher values than those corresponding to the virgin device. Finally, under the most stringent conditions, and after long stress time, a slight deceleration of the degradation, associated with a saturation of the generation of damage, is appreciated.

The classical explanation of the $I_{d}$ vs. $V_{g}$ characteristics degradation would be that this is due to both, trapping of electrons in the gate oxide $\left(\mathrm{N}_{\mathrm{of}}\right)$ and generation of acceptor-like interface states $\left(\mathrm{N}_{\mathrm{it}}\right)$. Nevertheless, since the registered transconductance increase cannot be explained merely by $\mathrm{N}_{\mathrm{of}}$ or $\mathrm{N}_{\mathrm{it}}$, a more particular degradation feature is expected to be responsible for this phenomenon. 


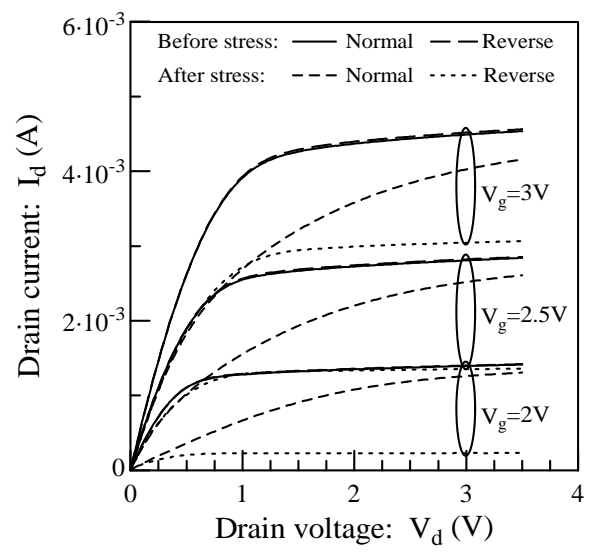

Fig. 3. $I_{d}$ vs. $V_{d}$ pre-stress and post-stress $(16000 \mathrm{~s}$, $\mathrm{V}_{\mathrm{d}}=6.5, \mathrm{~V}_{\mathrm{g}}=3.25$ ) characteristics for $\mathrm{V}_{\mathrm{g}}=2,2.5$ and $3 \mathrm{~V}$, measured in normal and reverse configurations.

\subsection{Localisation of the damage}

In view of this unusual hot-carrier degradation mode, output characteristics $\left(I_{d}\right.$ vs. $\left.V_{d}\right)$ were measured before and after stress experiments, both, interchanging (reverse) and without interchanging (normal) the source for the drain relative to the configuration in which the stress was performed. It was expected that if negative charge trapping occurred in a localised region close to the drain junction, their electrical effects must be masked by the drain depletion region.

A typical result is shown in Fig. 3. As expected, initial drain curves exhibit symmetry to the source-drain interchange. In contrast, after stressing the device, $I_{d}$ is lower, and a clear asymmetry between normal and reverse polarities appears.

As $V_{d}$ is increased, less difference is observed between the pre-stress and the post-stress $I_{d}$ vs. $V_{d}$ curves measured in normal configuration, showing a marked rounded shape. On the other hand, this phenomenon is not observed when source and drain are interchanged for the $I_{d}$ vs. $V_{d}$ measurements.

Both phenomena can be explained by negative charge trapping occurring in a limited region near the drain junction $[6,7]$. In this way, the $\mathrm{I}_{d}$ reduction in both configurations is attributed to $\mathrm{V}_{\text {th }}$ increase in the portion of the channel lying underneath the negative trapped charge. Moreover, the lack of symmetry indicates that the trapped charges are localised near the drain junction.

In normal configuration, the damage is located close to the drain and its electrical effects become more and more masked as the drain depletion region

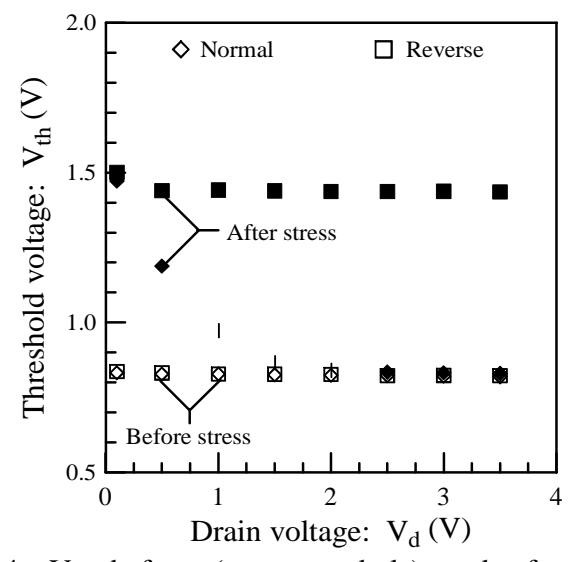

Fig. 4. $\mathrm{V}_{\text {th }}$ before (open symbols) and after (full symbols) stress $\left(18000 \mathrm{~s}, \mathrm{~V}_{\mathrm{d}}=6.5, \mathrm{~V}_{\mathrm{g}}=3.25\right)$ against $\mathrm{V}_{\mathrm{d}}$ in normal and reverse configurations.

widens. On the other hand, in reverse configuration, the damage is located close to the source junction and, independently of the applied $\mathrm{V}_{\mathrm{d}}$, it remains always electrically active.

An additional prove for the above model was obtained by measuring $I_{d}$ vs. $V_{g}$ characteristics in normal and reverse configurations applying increasing $V_{d}$ voltages. $V_{t h}$ results from such characteristics are shown in Fig. 4.

For low $\mathrm{V}_{\mathrm{d}}$ values, situation in which the channel of minority carriers spreads from the source to the drain (linear regime), no difference is appreciated between $\mathrm{V}_{\text {th }}$ values corresponding to normal and reverse configurations. However, as $\mathrm{V}_{\mathrm{d}}$ is increased, a marked asymmetry appears.

In the case of normal configuration, a clear $\mathrm{V}_{\text {th }}$ modulation associated to the widening of the drain depletion region is appreciated [11]. For sufficiently high $\mathrm{V}_{\mathrm{d}}\left(\mathrm{V}_{\mathrm{d}}>1.5 \mathrm{~V}\right)$, no significant $\mathrm{V}_{\text {th }}$ difference with respect to the pre-stress condition is registered, indicating that the damage has been totally shielded by the drain depletion region. In contrast, $V_{\text {th }}$ remains independent of $V_{d}$ in the case of reverse configuration, showing that the amount of electric active damage remains independent of $\mathrm{V}_{\mathrm{d}}$.

From the above results, as it should be expected, it can be drawn that the negative trapped charges are located in a limited region near the drain junction, which extends at most less than $\mathrm{L}_{\text {eff }} / 2$. Considering a simple approach relating applied $\mathrm{V}_{\mathrm{d}}$ with junction depletion width [12], from the results in Fig. 4, an estimation for the length of the damaged region $(\Delta \mathrm{L})$ was performed. These results showed that $\Delta \mathrm{L}$ was about the length of the pinchoff region $(\Delta L \approx 0.25 \mu \mathrm{m})$ [2]. 


\subsection{Effects on lateral channel electric field $\left(E_{\|}\right)$}

An additional characteristic feature of this hotcarrier degradation mode is the fact that substrate current ( $\left.\mathrm{I}_{\text {sub }}\right)$, or more precisely, multiplication factor $\left(\mathrm{I}_{\text {sub }} / \mathrm{I}_{\mathrm{d}}\right)$, has been found to monotonously rise as the stress progresses (Fig. 5). As it is well known, $I_{\text {sub }}$ is originated by the impact ionisation events taking place in the high field region near the drain junction, the simplest approach for $\mathrm{I}_{\text {sub }}$ is given by [2]:

$$
\frac{\mathrm{I}_{\text {sub }}}{\mathrm{I}_{\mathrm{d}}} \approx \mathrm{C} \cdot \mathrm{e}^{-\phi_{\mathrm{i}} / \mathrm{q} \lambda \mathrm{E}_{\mathrm{m}}}
$$

Being $E_{m}$ the maximum value of the lateral electric field $\left(\mathrm{E}_{\|}\right)$along the channel, $\mathrm{C}$ a weak function of $\mathrm{E}_{\mathrm{m}}$ and device parameters, $\lambda$ the hotelectron mean-free path and $\phi_{\mathrm{i}}$ the minimum energy that a hot-electron must have in order to create an electron/hole pair.

Thus, as no increase of $I_{d}$ is registered apart from that derived of the additional electrons from the impact ionisation events, a clear increase of $E_{m}$ occurs during the hot-carrier degradation of the devices. This $\mathrm{E}_{\mathrm{m}}$ increase is also attributed to the trapping of negative charges near the drain junction. In this situation, the trapped electrons reduce the effective $\mathrm{V}_{\mathrm{g}}$ in the damaged region, which results in an increase of the voltage drop and of $E_{\|}$near the drain junction $[6,13]$.

\section{Alternate static injection phases}

In order to investigate the connection between the registered transconductance and $\mathrm{E}_{\mathrm{m}}$ increases with the negative charge trapping phenomenon, some devices were first aged at $\mathrm{V}_{\mathrm{g}}=\mathrm{V}_{\mathrm{d}} / 2$ and then stressed under conditions which resulted in a holeinjection phase $\left(\mathrm{V}_{\mathrm{d}}\right.$ high, $\left.\mathrm{V}_{\mathrm{g}} \approx \mathrm{V}_{\mathrm{th}}\right)$ [3,9,14,15]. As expected, the hole-injection phase resulted in a partial neutralisation or compensation of negative trapped charges, and this allowed to prove the existence of a close relationship between the negative charge trapping and the transconductance and $\mathrm{E}_{\mathrm{m}}$ increases.

Fig. 6 shows $V_{\text {th }}, G_{\text {mmax }}$ and $I_{\text {sub }} / I_{d}$ stress time evolution for a device stressed at $\mathrm{V}_{\mathrm{d}}=6.5 \mathrm{~V}$ and at different consecutive $\mathrm{V}_{\mathrm{g}}$ voltages. From the figure, a basic correlation between these three parameters can be easily outlined. Under $\mathrm{V}_{\mathrm{g}}=\mathrm{V}_{\mathrm{d}} / 2$ stress bias conditions, positive $\Delta \mathrm{V}_{\mathrm{th}}$, together with $\mathrm{G}_{\mathrm{mmax}}$ and $\mathrm{I}_{\text {sub }} / \mathrm{I}_{\mathrm{d}}$ increases are registered. On the other hand, for

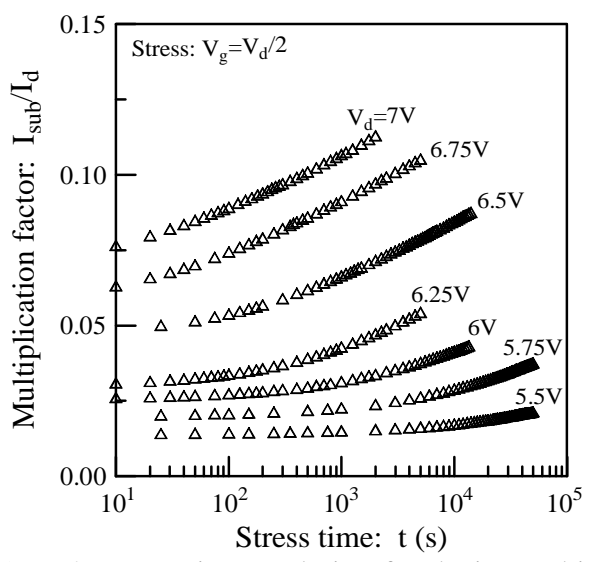

Fig. 5. $I_{\text {sub }} / I_{d}$ stress time evolution for devices subjected to different stress voltages.

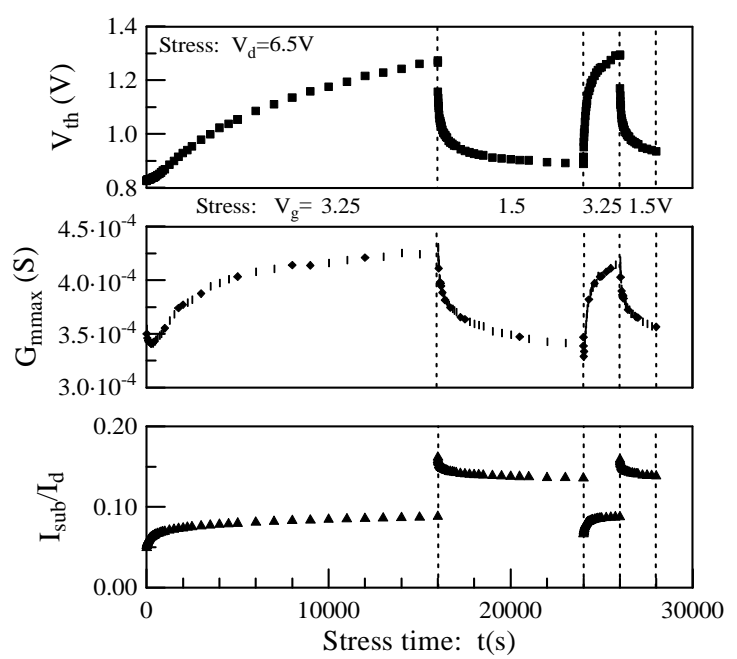

Fig. 6. $V_{\text {th }}, G_{\operatorname{mmax}}$ and $I_{d} / I_{\text {sub }}$ stress time evolution for a device stressed at $\mathrm{V}_{\mathrm{d}}=6.5 \mathrm{~V}$ and initially at $\mathrm{V}_{\mathrm{g}}=3.25 \mathrm{~V}$ and then at $\mathrm{V}_{\mathrm{g}}=1.5,3.25$ and $1.5 \mathrm{~V}$.

the hole-injection phases all these parameters are found to behave oppositely. In this way, $V_{t h}, G_{\operatorname{mmax}}$, and $\mathrm{I}_{\mathrm{sub}} / \mathrm{I}_{\mathrm{d}}$ after the third stress phase $\left(\mathrm{V}_{\mathrm{g}}=3.25 \mathrm{~V}\right)$ in Fig. 6 are similar to those registered after the first phase. A similar situation is observed after the second and fourth phases $\left(\mathrm{V}_{\mathrm{g}}=1.5 \mathrm{~V}\right)$.

\section{Two-piece analytical approach for $G_{m}$}

Having discussed in previous sections about nature and localisation of the damage and having also outlined a basic correlation between negative charge trapping and the $G_{m \max }$ increase, the ultimate reasons giving rise to this particular phenomenon can be now analysed. 
According to the model proposed by Haddara et al. [16], the channel of a degraded device can be considered, as a first approach, to be composed of two conductive regions of different conductance. In the linear region, the two corresponding resistances are added and the total channel conductance is given by:

$$
\begin{gathered}
\mathrm{G}=\frac{\mathrm{G}_{0} \bullet \mathrm{G}_{\mathrm{d}}}{\mathrm{G}_{0}+\mathrm{G}_{\mathrm{d}}} \\
\mathrm{G}_{0}=\frac{\mu \mathrm{WC}_{\mathrm{ox}}\left(\mathrm{V}_{\mathrm{g}}-\mathrm{V}_{\mathrm{th} 0}\right)}{\mathrm{L}-\Delta \mathrm{L}} \mathrm{G}_{\mathrm{d}}=\frac{\mu_{\mathrm{d}} \mathrm{WC}_{\mathrm{ox}}\left(\mathrm{V}_{\mathrm{g}}-\mathrm{V}_{\mathrm{thd}}\right)}{\Delta \mathrm{L}}
\end{gathered}
$$

being $\mathrm{G}_{0}$ the conductance of the L- $\Delta \mathrm{L}$ long defectfree region and $V_{\text {th0 }}$ and $\mu$ their threshold voltage and effective mobility, respectively. $G_{d}, V_{\text {thd }}$ and $\mu_{d}$ correspond to the damaged region of length $\Delta \mathrm{L}$ and width W.

From (2), the total transconductance of the degraded device $\left(\mathrm{G}_{\mathrm{m}} \equiv \mathrm{V}_{\mathrm{d}} \cdot \mathrm{dG} / \mathrm{dV}_{\mathrm{g}}\right)$ is given by:

$$
\mathrm{G}_{\mathrm{m}}=\frac{\mathrm{G}_{0}{ }^{2}}{\left(\mathrm{G}_{0}+\mathrm{G}_{\mathrm{d}}\right)^{2}} \mathrm{G}_{\mathrm{md}}+\frac{\mathrm{G}_{\mathrm{d}}{ }^{2}}{\left(\mathrm{G}_{0}+\mathrm{G}_{\mathrm{d}}\right)^{2}} \mathrm{G}_{\mathrm{m} 0}
$$

Where $\mathrm{G}_{\mathrm{md}} \equiv \mathrm{V}_{\mathrm{d}} \cdot \mathrm{dG}_{\mathrm{d}} / \mathrm{dV}_{\mathrm{g}}$ and $\mathrm{G}_{\mathrm{m} 0} \equiv \mathrm{V}_{\mathrm{d}} \cdot \mathrm{dG}_{0} / \mathrm{dV}_{\mathrm{g}}$ are the transconductances associated with the damaged and the defect-free portions of the channel, respectively.

According to (2) and (3), the total conductance and transconductance of the degraded transistor is equal to zero before the turn-on of the damaged region. Just after this turn-on point $\left(\mathrm{V}_{\mathrm{g}} \approx \mathrm{V}_{\text {thd }} \equiv \mathrm{V}_{\text {th } 0}+\Delta \mathrm{V}_{\text {th }}\right)$, the total transconductance is dominated by $G_{m d}$ term in (3), since $G_{d}$ is still very low whereas $G_{0}$ is much higher. In this situation, and because of the small $\Delta \mathrm{L} / \mathrm{L}$ ratio giving rise to $\mathrm{G}_{\mathrm{md}}>$ $\mathrm{G}_{\mathrm{m} 0}$, the total transconductance of the device can exceed the values registered before the stress. As $V_{g}$ is increased, $G_{d}$ rapidly surpasses $G_{0}$ and the effect of $\Delta \mathrm{V}_{\text {th }}$ in (3) becomes negligible. Consequently, the total transconductance $\left(\mathrm{G}_{\mathrm{m}}\right)$ decreases towards values similar to those of the fresh device.

\section{Bidimensional device electric simulation}

In order to better analyse the above effect on $\mathrm{G}_{\mathrm{mmax}}, 2 \mathrm{D}$ device electric simulations were carried out by means of TMA MEDICI, accordingly to previous results obtained from TMA SUPREM-3 process simulations [17].

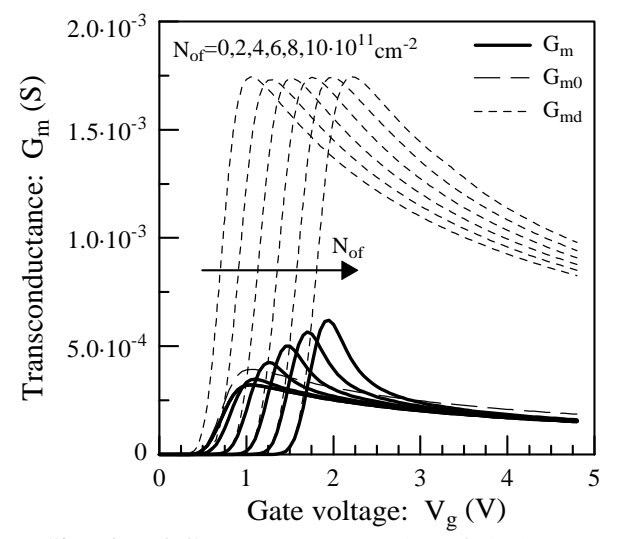

Fig. 7. Simulated $G_{m}$ vs. $V_{g}$ curves $\left(V_{d}=0.1 V\right)$.

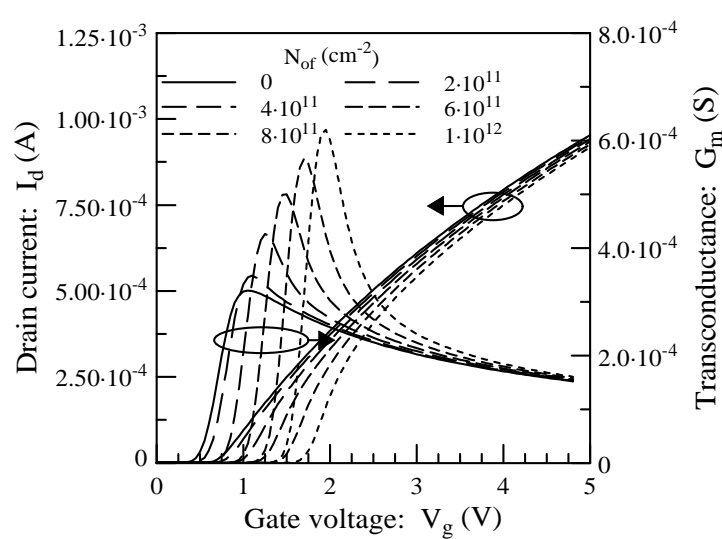

Fig. 8. Simulated $I_{d}$ vs. $V_{g}$ and corresponding $G_{m}$ from Fig. 7 results $\left(\mathrm{V}_{\mathrm{d}}=0.1 \mathrm{~V}\right)$.

The results from those studies are shown in Fig. 7 and Fig. 8. In this way, Fig. 7 shows transconductance $\left(\mathrm{G}_{\mathrm{m}}\right)$ vs. $\mathrm{V}_{\mathrm{g}}$ curves for six different simulations. The first one corresponds to a fresh device $\left(\mathrm{N}_{\mathrm{of}}=0 \mathrm{~cm}^{-2}\right)$ while the others correspond to a device in which different values of a spatial-uniform density of negative oxide trapped charges $\left(\mathrm{N}_{\mathrm{of}}=2\right.$ $10 \cdot 10^{11} \mathrm{~cm}^{-2}$ ) were placed at the $\mathrm{Si}-\mathrm{SiO}_{2}$ interface in a region $0.25 \mu \mathrm{m}$-long close to the drain. The simulated transconductances corresponding to each of the two different channel regions $\left(\mathrm{G}_{\mathrm{m} 0}\right.$ and $\left.\mathrm{G}_{\mathrm{md}}\right)$ are also plotted in Fig. 7. As it can be seen, just after the turn-on point, the total transconductance $\left(\mathrm{G}_{\mathrm{m}}\right)$ is clearly influenced by $G_{m d}$. However, as $V_{g}$ is increased, the total transconductance approaches that of the fresh device.

Finally, Fig. 8 shows $I_{d}$ and $G_{m}$ vs. $V_{g}$ simulated curves corresponding to Fig. 7 cases. As it can be seen, these simulation results qualitatively and quantitatively agree very well with the experimental degradation results (Fig. 1). So, the fact that the transconductance increases with $\mathrm{N}_{\text {of }}$ can 
(a)

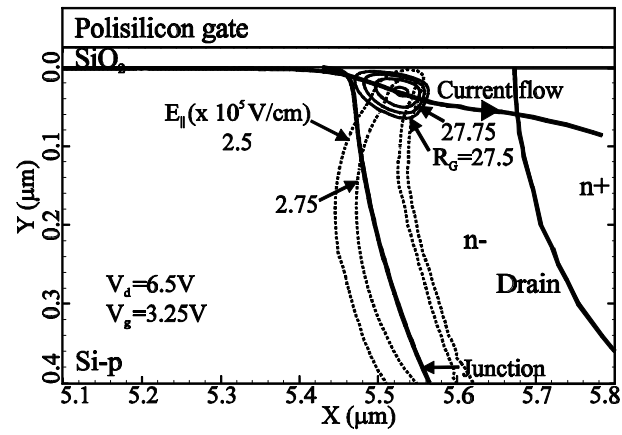

(b)

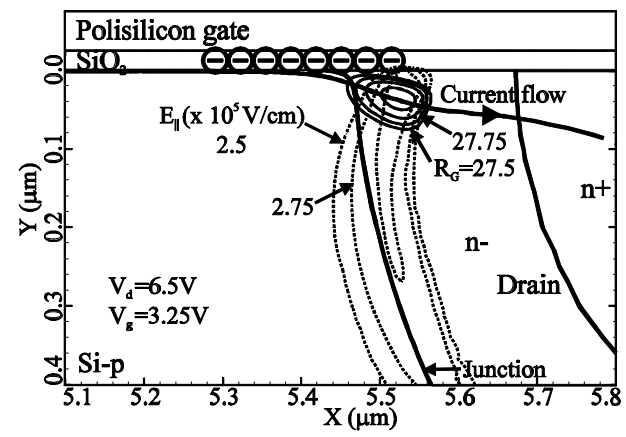

Fig. 9. Simulated distributions of $E_{\|}\left(0.25 \cdot 10^{5}\right.$ V/cmspaced contours) and current flow, together with impact ionisation contour lines $\left(\mathrm{R}_{\mathrm{G}}, 0.25\right.$-spaced contours), for (a) a defect-free device and (b) a device with $\mathrm{N}_{\mathrm{of}}=6 \cdot 10^{11} \mathrm{~cm}^{-2}$ near the drain junction.

be fully explained by means of the interaction between the damaged and the undamaged portions of the channel.

The above simulations were also analysed in terms of lateral channel electric field $\left(\mathrm{E}_{\|}\right)$and electron/hole pair generation rates. Following this trend, Fig. 9(a) shows contours of $E_{\|}$together with mean flow path and impact ionisation rate contours $\left(\mathrm{R}_{\mathrm{G}}, 10^{\mathrm{RG}}=\right.$ generated $\mathrm{e}^{-} / \mathrm{h}^{+}$pairs $\left./\left(\mathrm{cm}^{3} \cdot \mathrm{s}\right)\right)$ for a given polarisation $\left(\mathrm{V}_{\mathrm{ds}}=6.5 \mathrm{~V}, \mathrm{~V}_{\mathrm{gs}}=3.25 \mathrm{~V}\right)$ corresponding to a defect-free device.

Fig. 9(b) shows the same information but in this case corresponding to a device in which a spatial-uniform density of negative oxide trapped charges $\left(\mathrm{N}_{\text {of }}=6 \cdot 10^{11} \mathrm{~cm}^{-2}\right)$ was placed at the $\mathrm{Si}-\mathrm{SiO}_{2}$ interface in a region which extended $\Delta \mathrm{L}=0.25 \mu \mathrm{m}$ near the drain junction.

Comparing these two figures, one can easily notice that the presence of the negative trapped charges near the drain increases the lateral electric field and the impact ionisation rate. The magnitude of the $\mathrm{E}_{\|}$increase (about 10\%) is compatible with the measured $I_{\text {sub }} / I_{d}$ increase (Fig. 5 and Eq. (1)).

\section{Conclusions}

In this paper, an unusual hot-carrier degradation mode characterised by a transconductance increase during hot-carrier stressing of $\mathrm{n}$-channel MOS devices has been analysed. By means of studies of localisation and nature of the damage, this phenomenon is explained in terms of an exchange of the dominant role between the damaged and the undamaged regions of the channel. This degradation model is corroborated by $2 \mathrm{D}$ device electric simulation results.

\section{Acknowledgements}

This work has been partially supported by the Comisión Interministerial de Ciencia y Tecnología (CICYT), under project TIC95-1278-CE.

\section{References}

[1] Tsuchiya T, Kobayashi T, Nakajima S. IEEE Trans Electron Devices ED-34 (1987), pp 386-391.

[2] $\mathrm{Hu} \mathrm{C}$, et al. IEEE Trans Electron Devices ED-32 (1985), pp 375-385.

[3] Rafí J M, Campabadal F. Microelectronics Reliability 39 (1999), pp 869-874.

[4] Rafí J M, Campabadal F. Microelectronics Reliability 40 (2000), pp 743-746.

[5] Hsu F C, Tam S. IEEE Electron Device Lett EDL-5 (1984), pp 50-52.

[6] Schwerin A, Hänsch W, Weber W. IEEE Trans Electron Devices ED-34 (1987), pp 2493-2500.

[7] Roblin P, Samman A, Bibyk S. IEEE Trans Electron Devices (1988), pp 2229-2237.

[8] Hofmann K R, et al. IEEE Trans Electron Devices ED-32 (1985), pp 691-699.

[9] Doyle B S, et al. IEEE Trans Electron Devices 37 (1990), pp 1869-1876.

[10] Ono M, et al. IEEE Trans Electron Devices 42 (1995), pp 1510-1521.

[11] Chen K L, et al. IEEE Trans Electron Devices ED-32 (1985), pp 386-393.

[12] Doyle B, et al. IEEE Trans Electron Devices 37 (1990), pp 744-754.

[13] Nissan-Cohen Y, Franz G A, Kwasnik R F. IEEE Electron Device Lett EDL-7 (1986), pp 451-453.

[14] Tsuchiya T. IEEE Trans Electron Devices ED-34 (1987) 2291-2296.

[15] Heremans P, et al. IEEE Trans Electron Devices 35, (1988), pp 2194-2209.

[16] Haddara H, Cristoloveanu S. IEEE Trans Electron Devices ED-34 (1987), pp 378-385.

[17] SUPREM- $3^{\mathrm{TM}}$ and MEDICI ${ }^{\mathrm{TM}}$ were obtained from Technology Modeling Associates, Palo Alto, CA. 